\title{
Ion-beam-assisted deposition of molybdenum nitride films
}

\author{
E. P. Donovan and G. K. Hubler \\ Naval Research Laboratory, Code 6671, Washing1on, DC 20375 (USA)
}

\author{
M. S. Mudholkar and L. T. Thompson* \\ Department of Chemical Engineering, University of Michigan, Ann Arbor, MI 48109 (USA)
}

\begin{abstract}
A series of molybdenum nitride films was synthesized by electron beam evaporative deposition of molybdenum, with simultaneous bombardment by nitrogen ions from a Kaufman ion source. The nitrogen ions were accelerated to $500 \mathrm{or} 1000 \mathrm{eV}$. The film compositions and structures were determined using Rutherford backscattering spectrometry and X-ray diffraction respectively. Effective reflection and sputtering coefficients for nitrogen incident on the molybdenum nitride surfaces were extracted from the data. These coefficients were used to calibrate the deposition system and allowed the deposition of molybdenum nitride films with control of the nitrogen atom concentration to $\pm 2.3 \mathrm{at} \%$. In general, the films were polycrystalline with a high degree of texturing. The phases found in order of increasing measured nitrogen content were as follows: $\gamma-\mathrm{Mo}_{2} \mathrm{~N}$ (f.c.c.), $\beta-\mathrm{Mo}_{16} \mathrm{~N}_{7}$ (b.c.t.), B1-MoN (f.c.c.) then $\delta$-MoN (h.c.p.)
\end{abstract}

\section{Introduction}

The catalytic and sorptive properties of molybdenum nitrides are governed by their structures and compositions [1, 2]. Some effects of the bulk structure and composition on the catalytic and sorptive properties of molybdenum nitride powders have been reported [3] but little is known about the effects of the surface structure and composition. Our previous studies have shown that it is difficult to ascertain structure-function relationships for molybdenum nitride powders.

A detailed examination of the relationships between the surface structure and composition, and the catalytic function of molybdenum nitrides warrants the use of materials with well-defined surface structures and compositions. Structure and composition are very difficult to control using conventional synthesis techniques. However, ion-beam-assisted deposition (IBAD) offers greater control over the film composition and orientation than do other techniques $[4,5]$.

In this paper we discuss the synthesis of molybdenum nitride thin films using IBAD. We present the dependences of the stoichiometry and crystal structure on the arrival rate ratio of nitrogen to molybdenum and on the energy of the nitrogen ions. A deposition system calibration is detailed herein, including measurements of the effective sputtering and reflection coefficients for nitrogen incident on the films. The catalytic and sorptive properties will be reported elsewhere.

\footnotetext{
*Author to whom correspondence should be addressed.
}

\section{Experimental details}

After in situ sputter precleaning of the substrate with nitrogen ions to a dose of $7 \mathrm{mC} \mathrm{cm}^{-2}$, a series of films was deposited at temperatures less than $120^{\circ} \mathrm{C}$ onto substrates of area $1 \mathrm{~cm}^{2}$ cut from $\operatorname{Si}(100)$ wafers. Molybdenum was electron beam evaporated and deposited onto the substrate at a rate of $0.54 \mathrm{~nm} \mathrm{~s}^{-1}$, as measured by a quartz monitor. A space-chargeneutralized nitrogen ion beam from a Kaufman ion source $3 \mathrm{~cm}$ in diameter (discharge voltage, $50 \mathrm{~V}$; accelerator voltage, $-400 \mathrm{~V}$ ) impinged on the sample at an angle of $10^{\circ}$ from the surface normal. Most of the films were synthesized using a beam voltage of $500 \mathrm{~V}$. However, a few films were prepared using $1000 \mathrm{~V}$ to determine the effect of beam energy on the film properties. The distance from the ion source apertures to the substrate and to the Faraday cups (total entrance aperture area $0.146 \mathrm{~cm}^{2}$ ), which monitored the ion currents, was $20.5 \mathrm{~cm}$. The system base pressure was $2 \times 10^{-7}$ Torr. The operating pressure of $5 \times 10^{-5}$ Torr was maintained by means of mass flow control of the $\mathrm{N}_{2}$ gas. The previously measured charge state distribution of the ion beam [10] $\left(89 \% \mathrm{~N}_{2}^{+}\right.$and $\left.11 \% \mathrm{~N}^{+}\right)$resulted in the numbers of incident nitrogen atoms per ion being equal to 1.89 . For a beam voltage of $500 \mathrm{~V}$, on average, $94 \%$ of the atoms have energies of $250 \mathrm{eV}$. Films of uniform composition $200-300 \mathrm{~nm}$ in thickness were deposited by varying the nitrogen ion current while maintaining a constant molybdenum flux. A residual gas analyser (RGA) was used to monitor the gas composition during deposition. 
The samples were analysed for their nitrogen and molybdenum contents by means of Rutherford backscattering (RBS) using $\mathrm{He}^{2+}$ ions of energy $2 \mathrm{MeV}$ incident at an angle 15 from the beam direction. The crystal structure was determined by $\mathrm{X}$-ray diffraction using a Rigaku DMAX-B diffractometer. One sample was inserted into a cryostat and tested for the existence of a superconducting transition. This verified the presence of the B1-MoN phase, which could not be unambiguously identified by X-ray diffraction alone.

\section{Stoichiometric properties}

Figure 1 shows a typical RBS spectrum for a molybdenum nitride film deposited onto $\mathrm{Si}(100)$, and a single layer simulation using the analysis program RUMP [6]. The data were consistent with a film of uniform composition throughout the $300 \mathrm{~nm}$ thickness, assuming the film had bulk density.

Following analysis methods used in previous work [7], the incident nitrogen flux can be represented by

$F_{\mathrm{N}}=\delta(1+\beta) \gamma J / e$

Here, $\delta$ is 1.89 atoms $^{-1}{ }^{-1}$, as discussed previously, $(1+\beta)$ accounts for fast neutrals in the beam produced by charge exchange with ambient molecules, $\gamma$ is a geometric factor (1.05) to account for the differing positions of the Faraday cups and the sample, $J$ is the measured current density and $e$ is the electronic charge [8]. The charge exchange correction is assumed to be

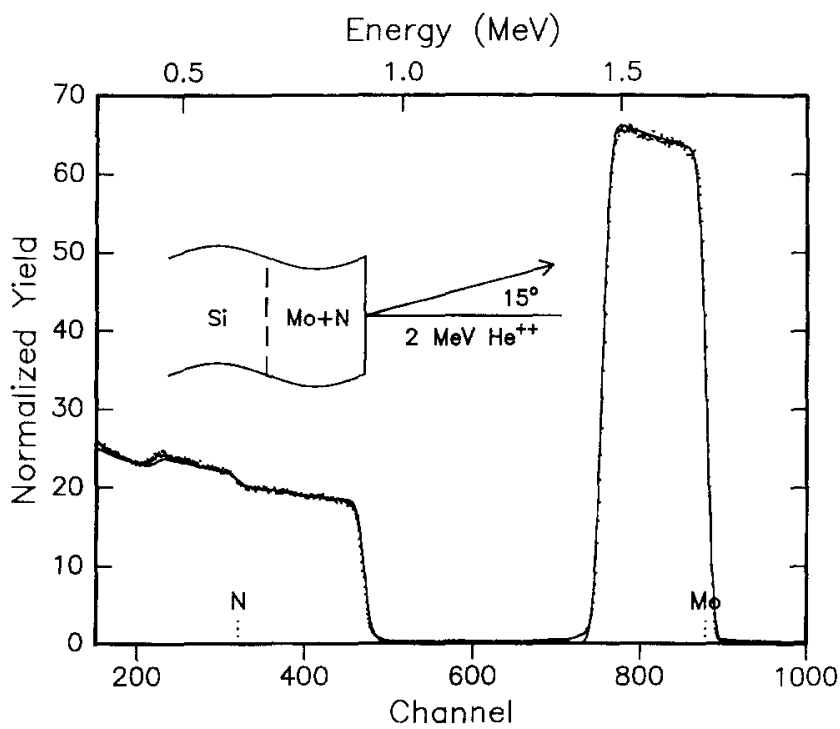

Fig. 1. Rutherford backscattering spectrum of molybdenum nitride film synthesized on silicon using IBAD with a beam of nitrogen ions of energy $500 \mathrm{eV}(\ldots)$. The line is a simulation using the analysis program RuMP. The film thickness and $\mathrm{N}$ : Mo stoichiometry were $1800 \times 10^{15} \mathrm{at} \mathrm{cm}^{-2}$ and 0.78 respectively. identical for all species in the beam and equal to 1.08 . which is correct for the reaction

$\mathrm{N}_{2}{ }^{+}(500 \mathrm{eV})+\mathrm{N}_{2} \rightarrow \mathrm{N}_{2}(500 \mathrm{eV})+\mathrm{N}_{2}{ }^{+}$

At the operating pressure of $5 \times 10^{-5}$ Torr, even if the charge exchange cross-sections differed by a factor of three for other interactions, such as $\mathrm{N}^{+}(500 \mathrm{eV})+\mathrm{N}_{2}$. the value of $(1+\beta)$ would change by less than $2 \%$.

The total measured areal densities of nitrogen and molybdenum, obtained by RBS, were divided by the deposition times to obtain the fluxes of nitrogen $\left(F_{N}^{\mathrm{l}}\right)$ and molybdenum $\left(F_{\mathrm{Mo}}^{\mathrm{f}}\right)$ incorporated into the film. These fluxes are plotted $\mathrm{c} s$. the incident nitrogen flux $F_{\mathrm{v}}$ in Figs. 2(a) (for nitrogen) and 2(b) (for molybdenum). The relationship between $F_{\mathrm{N}}^{\mathrm{f}}$ and $F_{\mathrm{N}}$ was very well described by a straight line with a slope of 0.45 and $y$ intercept of $0.43 \times 10^{15}$ at $\mathrm{cm}^{-2} \mathrm{~s}^{-1}\left(F_{\text {gas }}\right)$. The relationship between $F_{\mathrm{Mo}}^{\mathrm{f}}$ and $F_{\mathrm{N}}$ has been fitted to a straight line of slope -0.17 and $y$ intercept $3.4 \times 10^{15}$ at $\mathrm{cm}^{-2} \mathrm{~s}^{-1}\left(F_{\mathrm{Mo}}\right)$. The $v$ intercept $F_{M_{0}}$ agrees well with the evaporation rate of $0.54 \mathrm{~nm} \mathrm{~s}^{-1}$ multiplied by the bulk atomic density of
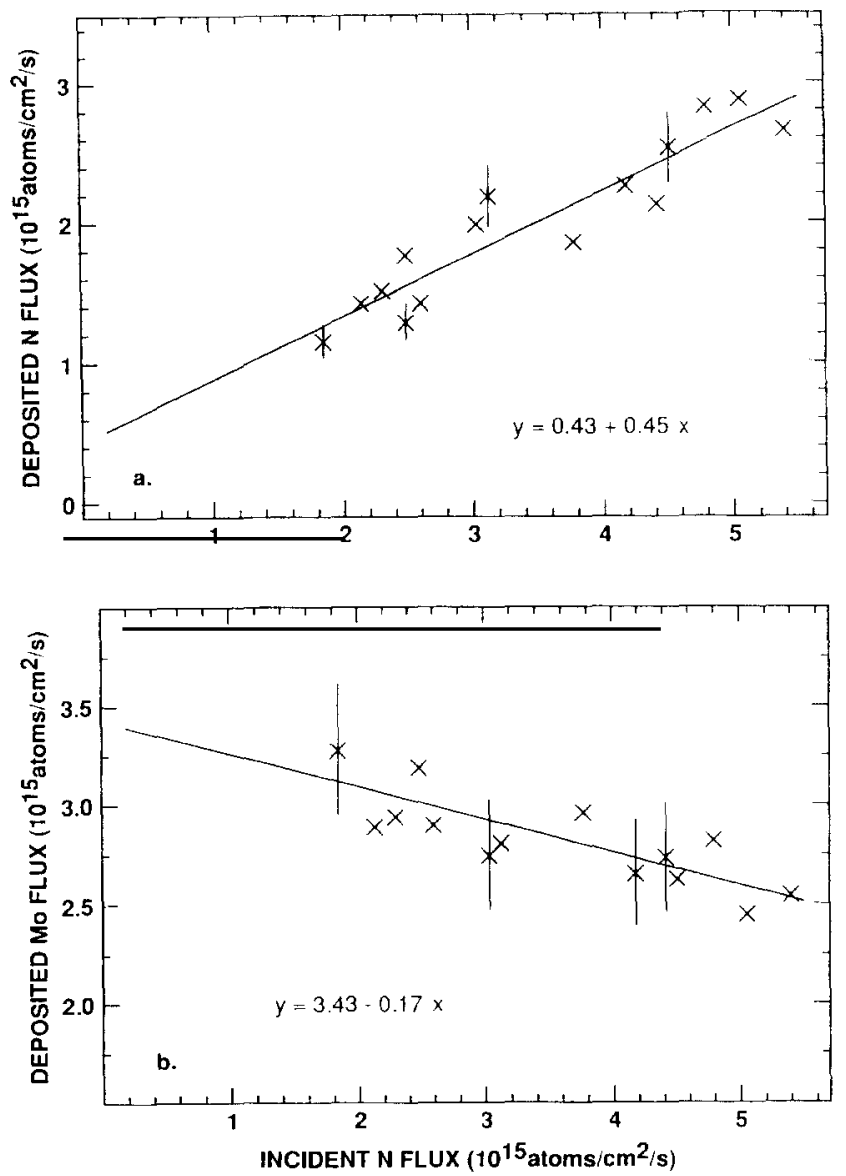

Fig. 2. Deposited flux of (a) nitrogen and (b) molybdenum as a function of the incident flux of nitrogen ions (mostly $\mathrm{N}_{2}{ }^{+}$) of energy $500 \mathrm{eV}:--$ fit; $\times$. RBS data. The equations in (a) and (b) are the results of a lincar fit to the data represented by the solid line. 
molybdenum, i.e. $6.4 \times 10^{22}$ at $\mathrm{cm}^{-3}$. The values of $F_{\text {gas }}$ and $F_{\mathrm{Mo}}$ indicate that 11 at. \% $\mathrm{Ni}$ was incorporated into the films through a process such as chemisorption of nitrogen from the gas phase.

The flux of nitrogen incorporated into the film (in Fig. 2(a)) has three components and can be modeled by

$F_{\mathrm{N}}^{\mathrm{f}}=F_{\mathrm{N}}\left(1-r-S_{\mathrm{N}}\right)+F_{\text {gas }}$

The term $r$ accounts for ions and energetic neutrals reflected from the surface. The term $S_{\mathrm{N}}$ accounts for sputtering of nitrogen previously incorporated into the surface. $F_{\text {gas }}$ then represents nitrogen incorporated into the film from the ambient $\mathrm{N}_{2}$ gas. These three terms should generally be considered as functions of the fraction of nitrogen at the surface. The nitrogen fraction is expected to vary with depth and remain near the surface value (from the data, this value is expected to be about 0.11 , at least for low values of $F_{\mathrm{N}}$ ) up to depths of the order of $R_{\mathrm{p}}$ minus one or two times $\Delta R_{\mathrm{p}}$. The nitrogen fraction should approach the bulk film value as the depth approaches $R_{\mathrm{p}}+\Delta R_{\mathrm{p}}$. The expected range and straggling are listed in Table 1 . Since $R_{\mathrm{p}}$ is approximately equal to $2 \Delta R_{\mathrm{p}}$ and from the stochastic nature of the stopping process, the value of the nitrogen fraction at the surface is expected to increase as $F_{\mathrm{N}}$ increases.

The nitrogen partial sputtering yield $S_{\mathrm{N}}$ should be a function of $x$, i.e. the nitrogen fraction, and, therefore, of $F_{\mathrm{N}}$ from three mechanisms.

(1) The effective sputtering yield $S$ is linearly dependent on the stopping power at the surface [8], which, for nitrogen ions in $\mathrm{Mo}_{1-x} \mathrm{~N}_{x}$, increases as $x$ increases.

(2) The projected range $R_{\mathrm{p}}$ of nitrogen decreases as $x$ increases. Therefore, the amount of nitrogen available to be sputtered from the surface will increase as $F_{\mathrm{N}}$ increases, faster than the increase discussed earlier. However, the decrease in $\Delta R_{\mathrm{p}}$ may have an opposing effect.

(3) The sputtering yield depends on the binding energy at the surface, which changes as the nitrogen fraction at the surface changes. We found no data on the dependence of the surface binding energies of molybdenum and nitrogen for molybdenum nitrides with changing atom fractions. The negative free energy of formation indicates that the binding energy increases with $x$; however, we expect this to be a weak effect, so we model the binding energy as a constant.

The reflection coefficient should decrease as $F_{\mathrm{N}}$ increases, since the average atomic mass decreases as $x$ increases at the surface. Finally, the gas absorption term $F_{\text {gas }}$ may depend on $F_{\mathrm{N}}$, since the beam can sputter adsorbed gases from the surface, alter the fraction of adsorption sites, and stimulate chemisorption of nitrogen in proportion to the beam current. These effects might compete with one another.

While the discussion in the previous paragraphs has suggested a non-linear dependence of $F_{\mathrm{N}}^{\mathrm{f}}$ on $F_{\mathrm{N}}$, the experimental data presented in Fig. 2(a) are adequately fitted by a straight line. This may indicate simply that the surface nitrogen fraction is not a strong function of $F_{\mathrm{N}}$. Therefore, we took the sum $r+S_{\mathrm{N}}$ as a constant equal to 0.55 . It should be recalled that $F_{\text {gas }}$ was found to equal $0.43 \times 10^{15}$ at $\mathrm{cm}^{-2} \mathrm{~s}^{-1}$.

The incorporated flux of molybdenum in the film in Fig. 2(b) is modeled with one term to account for the incident flux $\left(F_{\mathrm{Mo}}\right)$ and a second term to account for the flux sputtered away by the ion beam, i.e.

$F_{\mathrm{Mo}}^{\mathrm{f}}=F_{\mathrm{Mo}}-S_{\mathrm{Mo}} F_{\mathrm{N}}$

where $S_{\mathrm{Mo}}$ is the partial sputtering yield for nitrogen incident on $\mathrm{Mo}_{1-x} \mathrm{~N}_{x}$. Linear regression of the data presented in Fig. 2(b) gives a constant $S_{\mathrm{Mo}}=0.17$. We expected $S_{\mathrm{Mo}}$ to be a function of $F_{\mathrm{N}}$ for the same reasons given previously with respect to $S_{\mathrm{N}}$. The decrease in the concentration of molybdenum available for sputtering from the surface should decrease $S_{\text {Mo }}$, according to mechanism (2) described previously. Since the data are reasonably well represented by a straight line, we have assumed that $S_{\text {Mo }}$ was independent of $F_{\mathrm{N}}$ in the range investigated.

Table 1 compares the measured reflection and sputtering coefficients with those calculated using the computer code TRIM [9] for pure molybdenum and stoichiometric

TABLE 1. Calculated and experimental values of parameters used to calibrate the IBAD system for molybdenum nitride films

\begin{tabular}{|c|c|c|c|c|c|c|c|c|}
\hline $\begin{array}{l}E \\
\left(\mathrm{eV} \mathrm{at}{ }^{-1}\right)\end{array}$ & $\begin{array}{l}r \\
\text { (calc.) }\end{array}$ & $\begin{array}{l}S_{\mathrm{N}} \\
\text { (calc.) }\end{array}$ & $\begin{array}{l}S_{\mathrm{Mo}} \\
\text { (calc.) }\end{array}$ & $\begin{array}{l}r+S_{\mathrm{N}} \\
\text { (calc.) }\end{array}$ & $\begin{array}{l}S_{\mathrm{Mo}} \\
\text { (exp.) }\end{array}$ & $\begin{array}{l}r+S_{\mathrm{N}} \\
(\exp )\end{array}$ & $\begin{array}{l}R_{\mathrm{p}} \\
(\AA)\end{array}$ & $\begin{array}{l}\Delta R_{\mathrm{p}} \\
(\AA)\end{array}$ \\
\hline \multicolumn{9}{|l|}{$\mathrm{MoN}_{x}$} \\
\hline 250 & 0.21 & 0.18 & 0.08 & 0.39 & 0.17 & 0.55 & 8.9 & 4.5 \\
\hline 500 & 0.21 & 0.22 & 0.13 & 0.43 & 0.17 & 0.68 & 15 & 7.3 \\
\hline 1000 & 0.16 & 0.30 & 0.18 & 0.46 & - & 一 & 22 & 12 \\
\hline \multicolumn{9}{|c|}{ Molybdenum } \\
\hline 250 & 0.37 & 0.0 & 0.20 & 0.37 & - & - & 12 & 6.2 \\
\hline 500 & 0.30 & 0.0 & 0.36 & 0.30 & - & - & 18 & 9.0 \\
\hline 1000 & 0.30 & 0.0 & 0.53 & 0.30 & - & - & 28 & 15 \\
\hline
\end{tabular}


MoN. The calculated value of $r$ for nitrogen ions of energy $250 \mathrm{eV}$ decreases from 0.37 to 0.21 on going from molybdenum to molybdenum nitride, whereas $S_{\mathrm{N}}$ increases from 0 to 0.18 . To the extent that we can interpolate between the two data points, $r+S_{\mathrm{N}}$ is nearly constant at all $x$ values, although the calculated magnitude differs. However, the same calculations indicated that $S_{\mathrm{Mo}}$ for the nitrogen ions of energy $250 \mathrm{eV}$ should decrease from 0.20 to 0.08 as $x$ increases from 0 to 0.5 . which was not observed (Fig. 2(b)). The results show the correct trend with respect to the variation in $r+S_{N}$ with energy, but differ in magnitude. In addition, the results predict variations in $S_{\text {Mo }}$ at higher energies. If we extrapolate linearly the calculated values to $11 \mathrm{at} \% \mathrm{~N}$ at the surface, the agreement with the magnitudes improves over that achieved for MoN.

The measured nitrogen atom fraction $(\mathrm{N} /(\mathrm{N}+\mathrm{Mo}))$ $v s$. the measured ion current density is plotted in Fig. 3. The curve in this figure is derived from the straight line fits in Figs. 2(a) and 2(b). The experimental data points deviate from the fitted lines by \pm 2.3 at. $\% \mathrm{~N}$, indicating that the degree of control over the stoichiometry is quite good.

\section{Structural properties}

The molybdenum nitride phase that formed corresponded to the phase (equilibrium or metastable) with stoichiometry closest to the overall film composition. The $\gamma-\mathrm{Mo}_{2} \mathrm{~N}$ (f.c.c.) phase was predominant for films with nitrogen fractions between 0.26 and 0.34 (Fig. 4), while $\beta-\mathrm{Mo}_{16} \mathrm{~N}_{7}$ (b.c.t.) was predominant in films with nitrogen fractions between 0.33 and 0.43 . Between nitrogen fractions of 0.43 and 0.46 , the metastable B1-MoN (f.c.c.) phase was produced (Fig. 5). While standard data

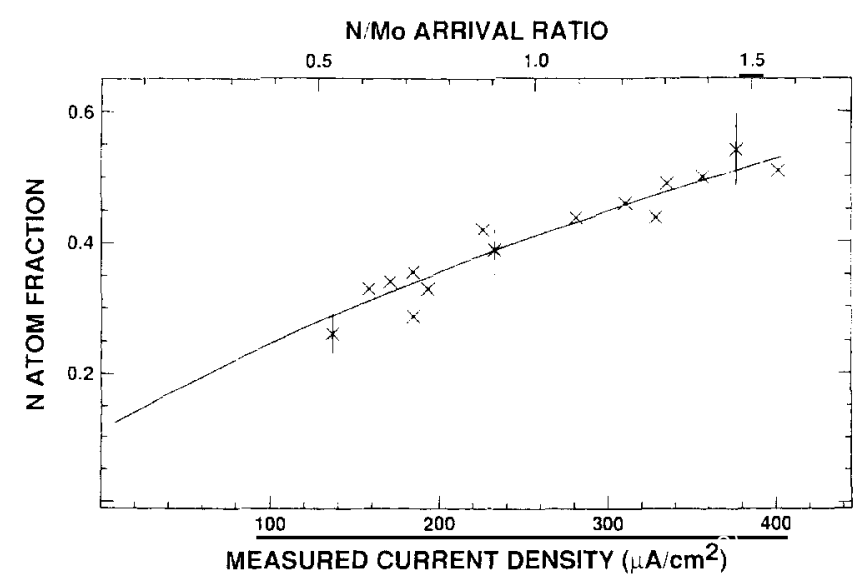

Fig. 3. Atomic fraction of nitrogen in the molybdenum nitride films is. the measured ion current density for nitrogen ions of energy $500 \mathrm{eV}$. The line is derived from the fit to the data presented in Figs. 2(a) and 2(b). while the X symbols represent RBS data.

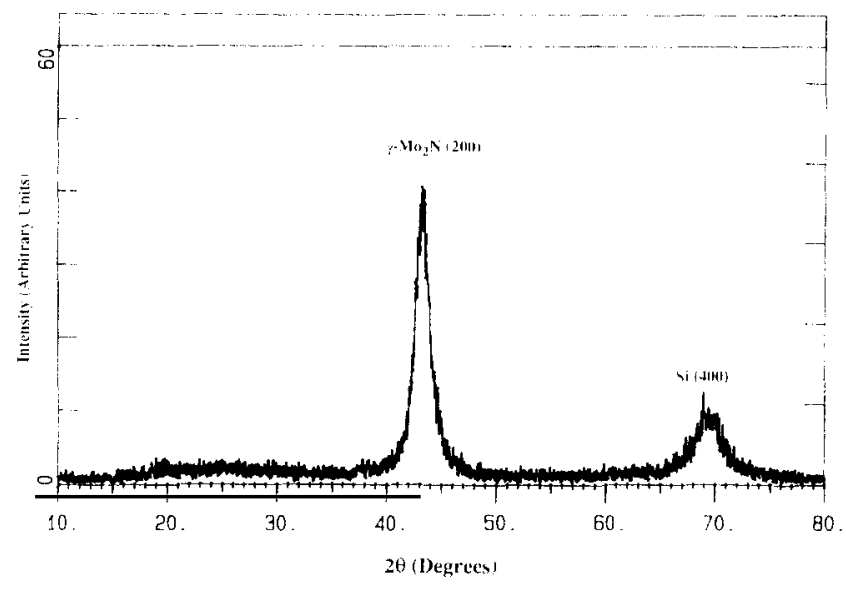

Fig. 4. X-ray diffraction pattern for an IBAD film consisting mostly of $y-\mathrm{Mo}_{2} \mathrm{~N}$. Strong texturing was observed with the $(200)$ planes oriented parallel to the surface. The beam energy was $500 \mathrm{eV}$.

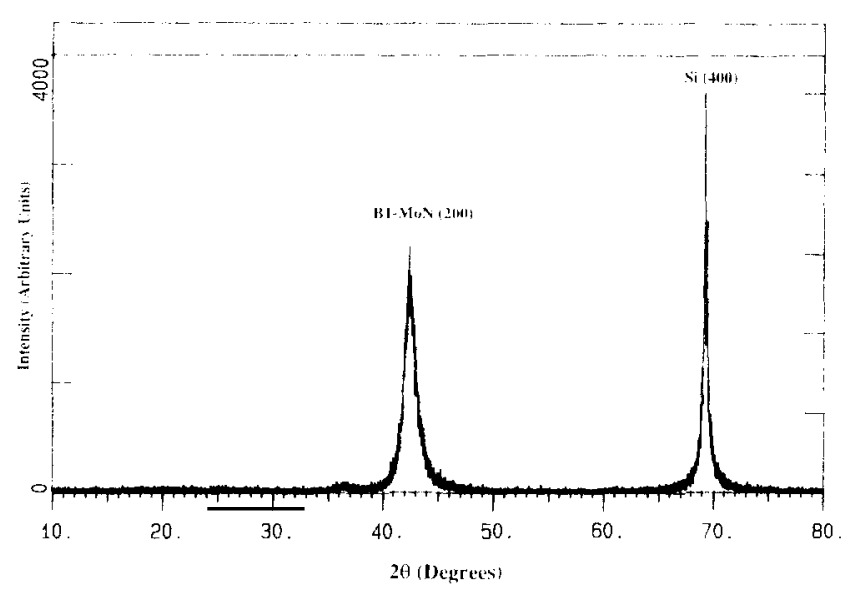

Fig. 5. X-ray diffraction pattern for an IBAD film consisting mostly of B1-MoN. Strong texturing was observed with the $(200)$ planes oriented parallel to the surface. The beam energy was $500 \mathrm{eV}$.

are currently unavailable for B1-MoN, the $d$ spacings observed for these films matched those predicted for B $1-\mathrm{MoN}[10]$. Films with nitrogen fractions between 0.46 and 0.5 contained a mixture of $\mathrm{B} 1-\mathrm{MoN}$ and $\delta$ MoN phases. For films with nitrogen fractions greater than $0.49, \delta$ - MoN (h.c.p.) was the predominant phase identified by $\mathrm{X}$-ray diffraction.

The film that we believe contained the B1-MoN phase was tested for evidence of a superconducting transition [11-14]. This film, with a stoichiometry of $\mathrm{Mo}_{0.55} \mathrm{~N}_{0.45}$. had a superconducting transition temperature of $5.8 \mathrm{~K}$ and a critical current density of $4 \times 10^{4} \mathrm{~A} \mathrm{~cm} 2$. This confirmed the presence of the $\mathrm{BI}-\mathrm{MON}$ phase in this sample.

The nitrogen ion energy indirectly affected the structure of the films. The nitrogen fraction measured in the films increased with increasing ion energy. Consequently, for films deposited at the same arrival rate ratio. a 
mixture of $\beta-\mathrm{Mo}_{16} \mathrm{~N}_{7}$ and $\mathrm{B} 1-\mathrm{MoN}$ phases was produced using nitrogen ions of energy $500 \mathrm{eV}$; however, $\delta$-MoN was predominant when ions of energy $1000 \mathrm{eV}$ were employed. Figure 6 summarizes the X-ray diffraction data.

Most of the films exhibited very strong texturing. The $\gamma-\mathrm{Mo}_{2} \mathrm{~N}$ crystallites were formed with the (200) planes preferentially oriented parallel to the substrate surface, and the $\beta-\mathrm{Mo}_{16} \mathrm{~N}_{7}$ crystallites were textured such that the (400) planes were parallel to the surface. The B1-MoN crystallites were oriented such that the $(200)$ planes were parallel to the substrate surface. When molybdenum was deposited in the absence of nitrogen ion bombardment, the planes of highest density, i.e. Mo(110), were preferentially oriented parallel to the substrate surface. This type of texturing, which is characteristic of vapor-deposited materials, was not observed for the IBAD molybdenum nitrides. The origin of texturing produced during IBAD has been the subject of considerable research [4-6]. In a few films deposited in a different deposition system, we found evidence that the texturing produced during IBAD depends on the angle of incidence of the ion beam [15]. We believe that the texturing is a result of ion channeling effects which favor the development of orientation with the more open directions aligned with the ion beam. Since channeling increases $R_{\mathrm{p}}$, this explanation is consistent with the lack of evidence for a strong dependence of the surface nitrogen fraction in the growing film on $F_{\mathrm{N}}$.

\section{Conclusions}

IBAD of molybdenum using a nitrogen ion beam at various nitrogen-to-molybdenum arrival ratios produced

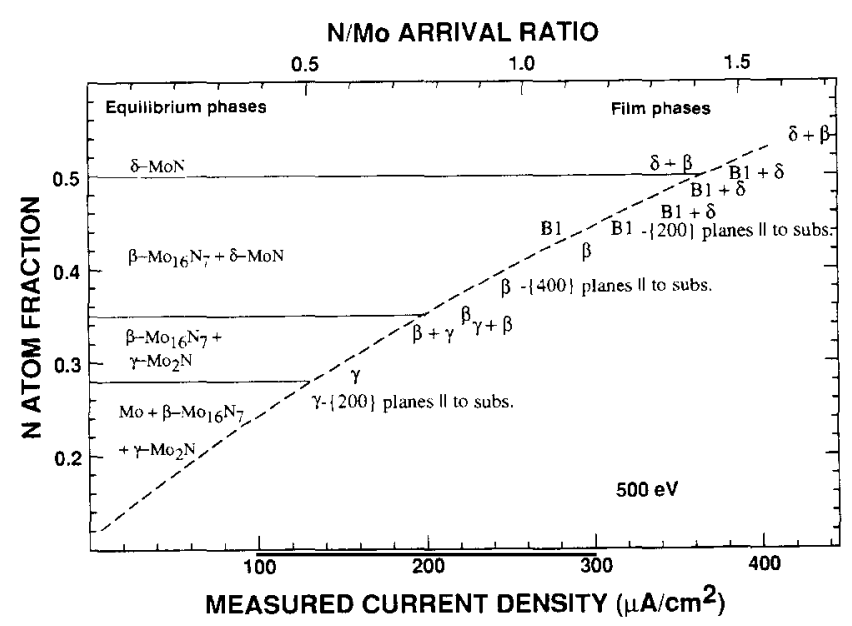

Fig. 6. Summary of film structures determined using X-ray diffraction. The dashed line indicates the model fit. The equilibrium phase diagram is represented on the left-hand side. The phases produced are indicated as a function of nitrogen-to-molybdenum arrival rate ratio and nitrogen atom fraction. molybdenum nitride films with $\mathrm{N}$ : Mo stoichiometries between 0.35 and 1.17. RBS data indicated that the films had uniform compositions. 11 at. $\% \mathrm{~N}$ was found to be directly incorporated into the growing film from the gas phase at a deposition pressure of $5 \times 10^{-5}$ Torr. The amount of additional nitrogen incorporated into the films was directly proportional to the ion current density. The fraction of nitrogen atoms reflected and sputtered from the films was measured and found to follow the same trend as Monte Carlo code predictions, but the agreement in absolute value was poor. The molybdenum sputtering coefficient for nitrogen incident on the molybdenum nitride surface was measured and the agreement with calculated values was excellent if the values were extrapolated to 11 at. $\% \mathrm{~N}$ at the surface.

The molybdenum nitride phase that formed corresponded to the phase with stoichiometry closest to the overall film composition. The $\beta-\mathrm{Mo}_{16} \mathrm{~N}_{7}$ and $\delta$-MoN phases were formed near the equilibrium compositions, but metastable phases were formed near nitrogen fractions of 0.3 and 0.5 , and consisted of $\gamma-\mathrm{Mo}_{2} \mathrm{~N}$ and B1-MoN phases respectively. X-ray diffraction evidence for the B1-MoN (f.c.c.) phase was confirmed by the existence of superconductivity in one of the films. Figure 6 summarizes the structural data.

There was a significant degree of texturing in the films. The $\gamma-\mathrm{Mo}_{2} \mathrm{~N}$ films were textured with the (200) planes oriented parallel to the substrate surface, while the $\beta-\mathrm{Mo}_{16} \mathrm{~N}_{7}$ films were oriented such that the (400) planes were parallel to the substrate surface. The results show that molybdenum nitride films with reproducible compositions and structures can be synthesized using a calibrated IBAD system.

\section{Acknowledgments}

The authors acknowledge financial support from the National Science Foundation (NSF CTS-8918107) and L. Soulen for measurement of the superconducting properties of the films.

\section{References}

1 J. C. Schlatter, S. T. Oyama, J. E. Metcalfe and J. M. Lambert, Ind. Eng. Chem. Res., 27 (1988) 1648.

2 H. Abe and A. T. Bell, Catal. Lett., 18 (1993) 9.

3 J-G. Choi, J. R. Brenner, C. W. Colling, B. G. Demczyk, J. L. Dunning and L. T. Thompson, Catal. Today, 15 (1992) 201.

4 J. K. Hirvonen, Mater. Sci. Rep., 6 (1991) 215.

5 F. A. Smidt, Int. Mater. Rev., 35(2) (1990) 61.

6 L. R. Doolittle, Nucl. Instrum. Methods B, 9 (1985) 344.

7 D. Van Vechten, G. K. Hubler, E. P. Donovan and F. D. Correll,

J. Vac. Sci. Technol. A, 8 (1990) 821 . 
G. K. Hubler, D. Van Vechten, E. P. Donovan and C. A. Carosella, I. Vac. Sci. Tedmol. A. \& $(1990) 831$.

8 R. Behrisch, Sputtering by Ion Bombardment, Springer, New York, 1981.

9 I. P. Biersack and L. G. Haggmark, Nut. Instrum. Methods. 174 (1980) 257

10 N. Terada. M. Naoe and Y. Hoshi. Ade. Cryo. Eng.. 32 (1986) 663.
11 D. A. Papaconstantopoulos and W. E. Pickett, Phrs Ret, B. 31 $11985) 7093$

12 N. Savvides, J. Appl. Phys, 62 (1987) 600.

13 G. Linker, H. Schmidt, C. Politis. R. Smithey and P. Ziemann, J. Phys. F, 16 (1986) 2167.

14 G. Linker. R. Smithey and O. Moyer, J. Phis. F. 14 1984) L.115.

15 M. S. Mudholkar and L. T. Thompson, unpublished. 1993. 